- 1

J.A-UR - 77-633

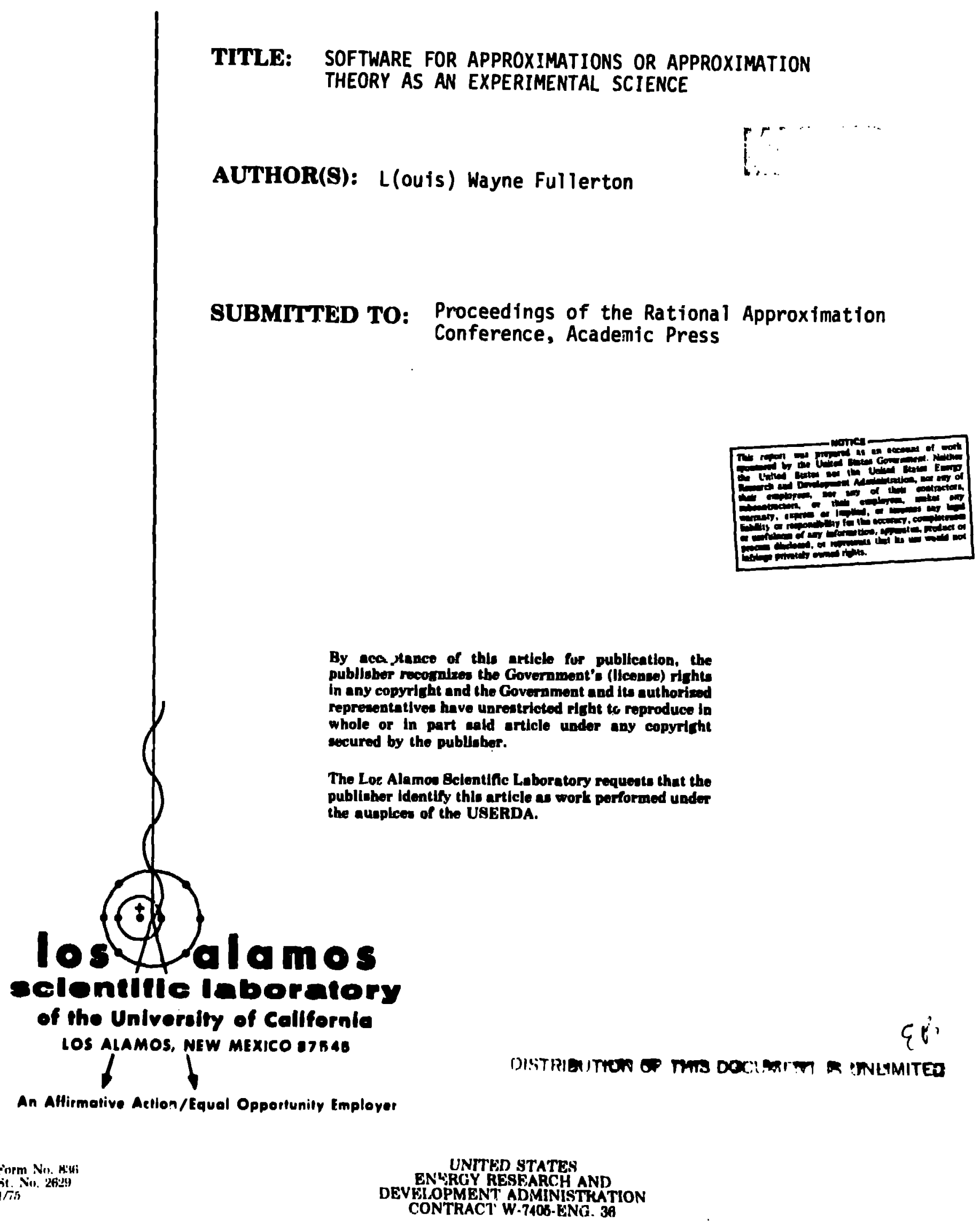




\section{SOFTWARE FOR APPROXIIATIONS \\ OR \\ APPROXIMATION THEORY AS AN EXPERIMENTAL SCIENCE}

\section{Wayne Fullerton}

Numerical analysis and approximation theory, in particular, can be an experimental science. This experimental nature is illustrated $w^{\prime}$ ith several more-or-less new results. In the first half of this paper techniques for estimating the accuracy and significance of approximations are given. In the second half several generalizations of Chebyshev series that lead to nearly best approximations with respect to almost arbitrary weight functions and basis sets are presented.

\section{Introduction}

Conversational references to the experimental nature of numerical analysis usually emphasize the trial-and-error aspects of research. Certainly I do not dispute the trial-and-error nature of numerical analysis research, but $I$ am most anxious to avoid 1 llustrating the errors $I$ have made. I call numerical analysis an experimental science in the same way that we all call physics or chemistry experimental sciences. There are two essential facets to an experimental science. First, theory or hypothesis suggests experiments that should be carried out. And second, experiments (conducted perhaps with computer programs) suggest new theoreticul results. I wish primarily to emphasize this latter facet. In the next section, it is shown how computational experience can dictate the kind of numerical analysis that should be donc. And in the third section, it is shown how experiments conducted with computor software can lead to new theoretical results. 


\section{Numerical Analysis for Software}

Anyone who has used an approximation program probably has been annoyed by its inability to detect user errors. In order to compute ar approximation, the user must supply function values that are somewhat more accurate than the approximation he desires. The more accurate values are often computed with a convenient ascending series for some argument values and an asymptotic series for other argument values. It is not uncommon to estimate incorrectiy the number of terms needed in one of the series, so that the two series fail to match to the required accuracy. Alternatively, the user may incorrectly estimate the stability against roundoff of one of the series, so that it is inaccurate even though enough terms are used.

Now when a user requests a very accurate approximation with inaccurate function values, some approximation programs will do a great deal of work and possibly fail to derive any approximation. Even if the user supplies accurate function values, his approximation form may be so unstable that the approximation (if it can be derived) is not useful. These common experiences with approximation software dictate that the troublesome situations be detected so that perplexed users can be warned.

\subsection{Input Function Accuracy}

We wish to assess the error of a user-supplied function. The general methods in this section may be used to derive, for example, the relative error but in this case Genuralized Chebyshev Series discussed in Section 3.2 must al so be used. Let us, therefore, restrict consideration to the estimation of absolute errors and simply note that extension of the results here to arbitrarily weighted errors is straight forward.

Suppose we compute a high-order Chebyshev series approximation to the user-supplied function. Fven though the series may contain 50 terins, only 10 terms may be significant. In such a case tice crror of the 10-term series would be nearly the same as 
the full 50-term scrics, and the magnitude of the last 40 terms would all be nearly the same. he can detertine how many terms are significant by observing that an $\mathrm{N}^{\text {th }}$ order series

$$
F(x)=\sum_{i=0}^{N} f_{i} T_{i}(x)
$$

is not only a near minimax approximation but also a discrete least squares approximation over the Chebyshev points $x_{j}=\cos \frac{j \pi}{N}$. Our strategy, then, is to estimate the number of terms to keep in the Chebyshev series in the same way that we estimate the number of terms to keep in any least squares approxImation (cf. Ralston [5]).

The sum of the squares of the errors for an $l$-th order series is

$$
v_{l}=\sum_{j=0}^{N}\left[F\left(x_{j}\right)-\sum_{i=0}^{\ell} f_{i} T_{i}\left(x_{j}\right)\right]^{2}
$$

If we ostimate the value of $F\left(x_{j}\right)$ by the $x^{\text {th }}$ order series and if we make use of orthogonality relations to eliminate cross produets, we obtain

$$
v_{l}=\sum_{j=0}^{N} \sum_{i=\ell+1}^{N} f_{i}^{2} T_{i}^{2}\left(x_{j}\right)=\frac{N+1}{2} \sum_{i=l+1}^{N} f_{i}^{2} .
$$

The standard error of one function value for an $l$-th order series is given by

$$
\sigma_{l}^{2}-\frac{v_{\ell}}{N-l}=\frac{N+1}{2} \frac{1}{N-l} \sum_{i=\ell+1}^{N} f_{1}^{2}
$$

he now compute these values for all 2 . In order to cvaluate the sum accuratcly, we start at $l=N$ for which the sum is zero and progressively decrease $\ell$. Next we check in a forward direction 
for some $\sigma_{k+1} \geq \sigma_{k}$. We then have an estimate of the number of terms, $k$, to keen and also an cstimate of the crror, $\sigma_{k}$, of the user-supplied function.

The scheme we have described can be used to detect both random errors and discontinuitles. The scheme works because we know the true function being approximated must have only very low-amplitude high "frequencies" and that it must have no discontinuities. Otherwise, a low-order polynomial approximation would be inappropriate. lie have found an efficient method for assessing the acciracy of input functions as well as output Chebyshev series appreximations. The requirement for such an accuracy estimate was dictated by computational expirience, and well known numerical techniques fortunately provided the solution.

\subsection{Stabllity of Approximation Form}

Knowing only the accuracy of an approximation is insufficlent, because the approxination may be unstable against roundoff. A ten-digit approximation is of Iittle use if 100-digit accuracy is needed to evaluate the approximation. A significance loss of 90 digits is, of course, uncontor; howcver, even a loss of one digit of significance may be unacceptable. Anyene who derives an approximation for use in a full machine-precision special function routine wil: be most distraught to learn the approximation is unstable against roundoff error while he is testing the special function routinu. He should be warned about the instabllity of the approximation when the approximation is derived. Once again, experience (or expertment) dictates the need for some numerical analysis rescarch. The results are just as casily obtained as in the previous subsection.

The significance loss incurred during the evaluation of an approximation can be easily estimated when the approximation 1tself is derived, provilled we do not try to do too much. A simple way of measuring the stabllity of an approximation is to calculate the numler of significant digits that should be kept in each 
of the coefficients of the approximation so that the extra crror introduced by rounding the cocfficients is no larger than the weighted error of the approximation. Because every major computer represents floating point nimbers with a nearly constant relative error, we need to calculate only one number, namely the number of significant digits to keep in ach coefficient.

Suppose now we are given an approximation

$$
A_{n}=\sum_{i=0}^{n} f_{i} \Phi_{i}(x)
$$

whose welghted error

$$
E=\max |\varepsilon(x)|=\max \left|w(x)\left[F(x)-A_{n}(x)\right]\right|
$$

is nearly minimax. We require the orthogonal functions $\phi_{i}$ to be normalized so that $w^{2}(x) \phi_{i}^{2}(x) \leq 1.0$ as in Section 3.2. In the special case $W(x) \equiv 1$, the $\phi_{1}$ are just Chebyshev polynomials. We have chosen to analyze orthogonal series, because they presumably are the most stable form and, moreovr $r$, the easiest form to derive.

Assume the errors introduced by arithmetic operations and by ovaluating the ${ }_{\text {: }}$ are ncgligible. Further assune the absolute error of the rounded incfficient $f_{i}$ is Gaussian distributed with standard deviation $\sigma_{1}$. Of course, the errors are not really Gaussian distributed, but we need only an estimate of the required significance. An error of 50 percent in our estimate corresponds to only 0.3 significant figures and is perfectly acceptable. The standard deviation of the absolute error of the approximation ovaluated with rounded coefficients is given by

$$
\sigma_{A}^{2}(x)=\sum_{i=0}^{n}\left(\frac{\partial A_{n}}{\partial f_{i}}\right)^{2} \sigma_{i}^{2} .
$$

Now 1 et $\delta$ be the standard deviation of the reiative error of . each rounded coefficient so that $o_{1}^{2}=f_{1}^{2} \delta^{2}$. Furthermore, 
recall that we want the weighted error introduced by the rounded coefficients to be less than the weighted error of the approximation, $\varepsilon$. Then we find

$$
\varepsilon^{2}=\max w^{2}(x) \sigma_{A}^{2}(x)=\max \left\{\sum_{i=0}^{n} f_{i}^{2} w^{2} \phi_{i}^{2}\right\} \delta^{2} .
$$

But the $\phi_{i}(x)$ are normalized so that $w^{2}(x) \phi_{i}^{2}(x) \leq 1$, and so

$$
\delta^{2} \geq \frac{\varepsilon^{2}}{\sum_{i=0}^{n} f_{i}^{2}}
$$

Finally, the number of significant figures, $S$, required to insure the effect of the rounding errors does not exceed the error of the approximation is

$$
s=-\log _{10} \delta
$$

Stable approximations are those for which $\delta$ is a large number compared with $\varepsilon$, that is, the required number of significant figures should be small. Thus, stabie approximations will have small leading coefficients -- the higher order coefficients are unimportant if the series converge reasonably quickly.

The extension of the analysis in this subsection to rational orthogonal series is straightforward, but the resulting expression for $\delta$ is not as elegantly simple as the result above.

\section{Software for Numerical Analysis}

In the previous section, the importance of numerical analysis applications to approximation programs used in a production mode was emphasized. Naturally, these programs become at the same time nore useful and reliahle as research tools. In this section, we emphasize the use of carcfully designed programs to -. conduct numerical experiments that may 1 ead to new theoretical 
results. Like a true experimental science, these theorctical results may immediately suggest new numerical experiments. Tho (almost) new theoretical results are used to illustrate the utility of computer programs as research tools in the next two subsections.

\subsection{Leveled Truncated Chebyshev Series}

Truncated Chebyshev series are well known to be nearly best absolute error approximations in the uniform norm. Because Chebyshev series are near minimax approximations and because they are quite stable against roundoff errors, it is natural to express true minimax approximations in terms of Chebyshev polynomials. It is also natural to wonder what the error of a minimax approximation looks like in terms of Chebyshev polynomials. The Chebyshev series of the error is almost trivially calculated, especially if one is already expressing minimax approximations in terms of Chebyshev polynomials.

Consider, therefore, the dominant error terms of a second order polynomial minimax approximation to the exponential function on the interval $[-1,+1]$ :

$$
\begin{gathered}
\varepsilon_{2}(x)=\ldots+.00013 \mathrm{~T}_{1}-.00553 \mathrm{~T}_{2} \\
+.04434 \mathrm{~T}_{3} \\
+.00547 \mathrm{~T}_{4}+.00054 \mathrm{~T}_{5}+\ldots
\end{gathered}
$$

The min error term is, as expected, $T_{3}$. Note, though, that the neighboring error terms are of tie same magnitude but opposite sign. If this happens only once or trice, it must be an accident. But it happens over and over. It even occurs for rational minimax approximations. Consider the Chebyshev series for the absolute error of a sccond order divided by a second order rational minimax approximation to the exponential: 


$$
\begin{aligned}
\varepsilon_{2,2}(x)=\ldots+ & .000009 \mathrm{~T}_{3}-.000038 \mathrm{~T}_{4} \\
& +.000067 \mathrm{~T}_{5} \\
& +.000037 \mathrm{~T}_{6}+.000011 \mathrm{~T}_{7}+\ldots
\end{aligned}
$$

As anticipated, $T_{5}$ is the dominant error term. And again neighboring error terms are of the same magnitude but opposite sign. Because the behavior we observe for these two cases occurs very frequently, we should consider an explanation.

A truncated Chebyshev series is ironically guaranteed to have a nonuniform error curve. If, for example, we truncate a Chebyshev series at fourth order, then the dominant error term will ordinarily be $T_{5}$. The next error term will be $T_{6}$, and this error term (if nonzero) will constructively interfere with $\mathrm{T}_{5}$ in some places and destructively interfere in other places. We truncate a Chebyshev series to obtain a nearly best approximation, but at the same time we insure the exror curve is ronuniform.

From the above numerical results we know what to do about the interference of higher order error terms with the dominant error term: we modify the truncated Chebyshev series so that lower order error terms of the same magnitude but opposite sign are introduced in the error expansion. This procedure works because the sum of the high and low order terms have zeroes exactly where the dominant error term has extremae. To see this effect, make the transformation $x=\cos \theta$. The dominant error term is then $T_{m}(x)=\cos m \theta$, and furthermore

$$
\begin{aligned}
T_{m-l}(x)-T_{m+l}(x) & =\cos (m-l) \theta-\cos (m+l) \theta \\
& =2 \sin l \theta \quad \sin m \theta .
\end{aligned}
$$

The nonzero low order error term aliases the high order error term and, therefore, reduces interference effects.

We have, then, derived a technique for leveling truncated Chebyshev series - - a technique sugyested solely by Chebyshev 
series expansions of true minimax approximation errors. The leveled Chebyshev series should be regarded only as first order modifications to truncated Chebyshev series, because the "ntroduction of the lower order crror terms simply avoids the addition of more error at the extremae of the main error term. Nonetheless, the improvement is obtained at essentially no cost, and wile a truncated Chebyshev series may deviate from a minimax approximation by perhaps 20 or 30 percent, the deviation of a leveled Chebyshev serics is more likely to be only a few percent. Economization of a power series [2] is a commonly employed method of obtaining a good approximation from a power series. In effect, the polier series is converted to a Chebyshev series, then the small amplitude high order terms are dropped. One then obtains an economical approximation with fewer terms, but with little additional error. The results in this subsection could, however, be used to obtain a still better approximation with the same numisr of terms. Rather than truncating the Chebyshev series, the Chebyshev series should be leveled.

\subsection{Generalized Chebyshev Series}

Truncated Che'yshev series are nearly best approximations in the uniform norm. Unfortunately, they are only nearly best polynomial approximations and only in the sense of absolute error. It is natural to wonder about generalizations that would be good for arbitrary weight funciions and non-polynomial bases. Originally this problem was motivated by the need for good starting values for the rational Remez iteration. However, before the rational problem is studied, we should soive the polynomiai case. Consider first the problem of finding an approximation $A_{n}(x)$ to the function $F(x)$ on $[-1,+1]$ with weight function $W(x)=1$, such that the error

$$
\varepsilon(x)=W(x)\left[F(x)-A_{n}(x)\right]
$$

is near minimax. He know, of course, the solution is first to 
define some polynomials -- Chebyshev polynomials -- from the orthogonality condition

$$
\int_{-1}^{1} \frac{T_{m}(x) T_{n}(x)}{\sqrt{1-x^{2}}} d x=0, m \neq n,
$$

with the $T_{n}(x)$ normalized so that their extreme value is unity. Next we expand $F(x)$ in a series

with

$$
F(x)=\sum f_{i} T_{i}(x)
$$

$$
f_{i}=\frac{1}{h_{n}} \int_{-1}^{1} \frac{F(x) T_{i}(x)}{\sqrt{1-x^{2}}} d x
$$

where

$$
h_{n}=\int_{-1}^{1} \frac{T_{i}^{2}(x)}{\sqrt{1-x^{2}}} d x
$$

When this series is truncated at $n-t h$ order, we obtain the desired approximation $A_{n}$.

In generalizing to arbitrary weights, it is reasonable to suppose a simple function of the weight must be included in the orthogonality condition. I incorrectly conjectured that the weight in the orthogonality condition might be $w(x) / \sqrt{1-x^{2}}$ or perhaps $\sqrt{1+(x)} / \sqrt{1-{ }^{2}}$. The problem of finding the appropriate orthogonal polynomials and expansion coefficients can be posed essentially as a Gauss-Christoffel quadrature problem. Because a good Gauss-Christ offel quadrature program was availatle to me, I quickly learned that these conjectures did not lead to nearly best approximations. I did observe, however, that the quadrature weight containing $\sqrt{(x)}$ was the worse, so I tried 
$h^{2}(x) / \sqrt{1-x^{2}}$. That choice I found to be the correct one. Yon that the correct generalization is known, it is easy - embarrassingly easy - to cxplain why.

he will be expanding $F(x)$ in a series of some orthogonal polymomials

$$
F(x)=\sum f_{i} \phi_{i}(x)
$$

and when we truncatc the series at $n$-th order, the weighted error will be roughly $H(x) \phi_{n+1}(x)$. We want this error to be an equal ripple curve, just like $T_{n+1}(x)$ would bc. Thus, the analogue of the Chebyshev polynomial $T_{i}$ is $W^{\prime} \phi_{i}$. And when we substitute this result in the orthogonaljty condition, we find the $\phi_{i}$ are given by

$$
\int_{-1}^{1} \frac{h^{2}(x) \phi_{m}(x) \phi_{n}(x)}{\sqrt{1-x^{2}}} d x=0, m \neq n
$$

See Gautschi [4] for a discussion of the derivation of orthogonal polynomials. Nie choose to normalize these polynomials so that the extremum of $H(x) \phi_{i}(x)$ is unity. Such a normalization allows one to assess readily the accurac: of a truncated series in these polynomials. The weighted error bound is simply the sum of the absolute values of all the coefficients dropped from the series, and this bound is usually close to the true weighted crror. Truncated generalized Chebyshev series often are within 20 or 30 percent of the corresponding true weighted minimax approximations. Because each approximation will usually have a unique weight function, the use of a general Gauss-Christoffel quadrature routine is not the best way to cbtain the orthogonal polynomials and expansion coefficients. The integrals needed can be done efficiently by an automated Gauss-Chebyshev scheme, and the cxpansion coefficients can be derived at the same time as the recurrence coefficients for the orthogonal polynomials. 
Generalization to non-polynomial bases is now straightfor:ard, in principle. Instcad of constructing the orthogonal functions $\phi_{i}$ from the basis $x^{i}$, it is necessary to orthogonalize the desired basis functions. In practice, the integrals are not so easily evaluated, and one must be certain that the basis functions form a Chebyshev set. For a brief discussion of the applications of these approximations see fullerton [3].

Further generalization to orthogonal-Padé approximations, where we require

$$
\frac{\sum_{i=0}^{m} p_{i} \phi_{i}}{\sum_{i=0}^{n} q_{i} \phi_{i}}=\sum_{i=0}^{m+n} f_{i} \phi_{i}(x),
$$

with $q_{0} \equiv 1$, are now casily obtained. We first discretize at the zeroes of $\phi_{m+n+1}(x)$, and solve the resulting linear equations for the $p_{i}$ and $q_{i}$. As with Chebyshev-Padé approximations [1], we obtain either a degenerate approximation or a good one. If it is degenerate, we are not interested in the approximation because the next lowest order rational approximation wiil be nearly as good as the one we are trying to derive. If the discretized approximation is good, we can then use it as a starting value for finding differential corrections to the rational coefficients in the above nonlinear equation. The discretized solution usually is so close to the orthogonal-Pade solution, that only one or two iterations of the linearized version of the above equation are neccssary. The orthogonal-pade approximations always secm to be more accurate than the corresponding discretized approximations. N1though the solution for orthogonal-pade approximations outlined here is not nearly as elegant as the solution for Chebysher-Padé approximations given by Clenshaw and lord [1], at least we have obtained a solution. 
4. Conclusions and Acknowiledgements

The need for quality software dictates to some extent the type of numerical analysis that should be done, and several examples were given in Section 2. The results there led to programs that were not only much more uscful production tools, but also much more useful research tools. Employing computer programs to conduct numerical experiments was shown in Section 3 to be an effective means of testing conjectures and deriving new theoretical results. I an confident that I would never have obtained the results in Section 3 unless I had had quality software tools available. Approximation theory and numerical analysis can be an experimental science.

Dr. D. D. Warner suggested the solution to the input function accuracy probiem given in Section 2.1, and I am grateful to him for the suggestion. I am also grateful to Warner for numerous, long conversations that certainly led to some of the other results presented in this paper.

\section{References}

1. Clenshaw, C. W. and K. Lord, Rational approximations from Chebyshev serios, Studies in Numerical Analysis (B. K. P. Scaife, editor), Acadeluic Press, Londun, 1974, pp. 95-113.

2. Dahlquist, G. and A. Björck, Numerical Methods, Prentice Hail, Fingl ewood Cilffs, N. J., 1974, pp 125-126, (Translated by N. Anderson).

3. Fullerton, L. W., Portable special function routines, Proc. Workshop on Portability of Numerical Software, (W. CoweI1, editor), Springer - Ver1ag, New York, (in Press).

4. Gautschi, li., Construction of Gauss-Christ offel quadrature formulae, Math. Comp., 22 (1968), ap. 251-270.

5. Ralston, A., A First Course in Numerical Analysis, McGraw H111, New York, 19!65, Pp. 334-235.

L. W. Fullerton

Group C3, MS 265

Los Alamos Scientific Laboratory

Los Alamos. New Mexico 87545 